Rev. Inst. Flor. v. 29 n. 1 p. $57-69$ jun. 2017

http://dx.doi.org/10.24278/2178-5031.201729104

ISSN impresso 0103-2674/on-line 2178-5031

\title{
INFLUÊNCIA DA ADUBAÇÃO NPK NO CRESCIMENTO INICIAL DE Tectona grandis L.f. EM CAMPO ${ }^{1}$
}

\section{INFLUENCE OF NPK FERTILIZATION ON INITIAL GROWTH OF Tectona grandis L.f. IN FIELD}

\author{
Cristiane Ramos VIEIRA², ${ }^{2}$; Oscarlina Lúcia dos Santos WEBER ${ }^{3}$; José Fernando SCARAMUZZA ${ }^{3}$
}

RESUMO - Com relação às espécies florestais, ainda há muito que estudar sobre a adubação no campo. Diante disso, instalou-se um experimento com o objetivo de analisar o crescimento das mudas de Tectona grandis L. f. no campo, submetidas a diferentes adubações com NPK. Primeiramente, efetuou-se o crescimento das mudas no viveiro, para isto, realizou-se a calagem do solo e, após 15 dias, a adubação, utilizando-se sacolas plásticas. Essas adubações se basearam nos níveis: $\mathrm{N}=0,40,80$ e $120 \mathrm{~kg} \mathrm{ha}^{-1} ; \mathrm{P}_{2} \mathrm{O}_{5}=0,50,100$ e $200 \mathrm{~kg} \mathrm{ha}^{-1} \mathrm{e}$ $\mathrm{K}_{2} \mathrm{O}=0,50,100$ e $200 \mathrm{~kg} \mathrm{ha}^{-1}$. Após 150 dias, as plantas dos 20 tratamentos que apresentaram os maiores crescimentos em altura e diâmetro foram transplantadas para o campo. No campo, o solo foi previamente calcareado e adubado e as mudas foram distribuídas em blocos casualizados com seis repetições. $\mathrm{O}$ crescimento foi analisado periodicamente aos 3, 6, 9 e 12 meses. Após 12 meses, as plantas foram retiradas e submetidas às análises de biomassa seca e macro e micronutrientes. $\mathrm{O}$ crescimento de $T$. grandis foi influenciado pela adubação com NPK, sendo que a maior influência de $\mathrm{K}$ foi observada no crescimento em altura. As doses recomendadas para o crescimento inicial no campo foram $40 \mathrm{~kg} \mathrm{ha}^{-1}$ de $\mathrm{N}$, $50 \mathrm{~kg} \mathrm{ha}^{-1}$ de $\mathrm{P}_{2} \mathrm{O}_{5}$ e de 50 ou $100 \mathrm{~kg} \mathrm{ha}^{-1}$ de $\mathrm{K}_{2} \mathrm{O}$.

Palavras-chave: Tectona grandis; adubo mineral; solos florestais; nutrição florestal.

\begin{abstract}
With respect to forest species, there is still much study about field. For this, an experiment was carried out to analyze the growth of Tectona grandis L. f. in the field, submitted to different fertilizations with NPK. First, the seedlings were grown in the nursery, for this purpose, the soil was limed and, after 15 days, fertilized in plastic bags. These fertilizations were based on levels: $\mathrm{N}=0,40,80$ and $120 \mathrm{~kg} \mathrm{ha}^{-1}$; $\mathrm{P}_{2} \mathrm{O}_{5}=0,50,100$ and $200 \mathrm{~kg} \mathrm{ha}^{-1}$, and $\mathrm{K}_{2} \mathrm{O}=0,50,100$ and $200 \mathrm{~kg} \mathrm{ha}^{-1}$. After 150 days the plants of the 20 treatments that presented the greatest growth in height and diameter were transplanted to the field. In the field, the soil was previously limed and fertilized, and the seedlings were distributed in randomized blocks with six replicates. Growth was analyzed periodically at 3, 6, 9 and 12 months. After 12 months, the plants were removed and submitted to analyze of dry biomass, macro and micronutrients. T. grandis growth was influenced by the fertilization with NPK, and the greater influence of K was observed in the height growth. The recommended doses to initial growth in the field were $40 \mathrm{~kg} \mathrm{ha}^{-1}$ of N, $50 \mathrm{~kg} \mathrm{ha}^{-1}$ of $\mathrm{P}_{2} \mathrm{O}_{5}$ and 50 or $100 \mathrm{~kg} \mathrm{ha}^{-1}$ of $\mathrm{K}_{2} \mathrm{O}$.
\end{abstract}

Keywords: Tectona grandis; mineral fertilizer; forest soil; forest nutrition.

\footnotetext{
${ }^{1}$ Recebido para análise em 12.03.2017. Aceito para publicação em 08.05.2017.

2Universidade de Cuiabá, Curso de Agronomia, Av. Beira Rio, 3100, Bairro Jardim Europa, 78065-443, Cuiabá, MT, Brasil.

${ }^{3}$ Universidade Federal de Mato Grosso, Departamento de Solos e Engenharia Rural, Av. Fernando Corrêa da Costa, n. 2367, Bairro Boa Esperança, 78060-900, Cuiabá, MT, Brasil.

${ }^{4}$ Autor para correspondência: Cristiane Ramos Vieira - cris00986@hotmail.com
} 


\section{INTRODUÇÃO}

Os solos sob Cerrado são, em geral, de elevada acidez, altos teores de Al trocável, baixa disponibilidade de cátions básicos como $\mathrm{Ca}$ e $\mathrm{Mg}$, e de baixa disponibilidade de $\mathrm{P}$ na forma disponível para as plantas. Características que comprometem o crescimento das mesmas e, consequentemente, sua produtividade. Dessa maneira, práticas silviculturais como a calagem e a adubação com NPK tornam-se indispensáveis quando se trata de plantios em área de Cerrado.

Porém, dos estudos a respeito da adubação de espécies nativas, grande parte está relacionada apenas à etapa de viveiro, pois se trata de dados que podem ser mais rapidamente e facilmente obtidos. O que aumenta a necessidade de obter informações a respeito das necessidades das mudas após o plantio, pois, a falta de conhecimento nesta etapa compromete o produto final da espécie, principalmente quando se trata de madeira.

Tectona grandis, vulgarmente conhecida como teca, é uma árvore de grande porte da família Verbenaceae, nativa das florestas tropicais do Sudeste asiático e atualmente cultivada nos países tropicais. No ano de 2011, de acordo com a Associação Brasileira de Produtores de Florestas Plantadas - ABRAF, a área total de plantios da espécie, no Brasil, era de 65.440 hectares, distribuídos entre os estados do Acre, Amapá, Mato Grosso do Sul, Paraná, Rondônia, São Paulo, Tocantins e, principalmente, Mato Grosso e Pará (ABRAF, 2012). De acordo com Pelissari et al. (2013), o estado do Mato Grosso tem as maiores áreas plantadas (cerca de 50 mil hectares). Apresentando desenvolvimento quantitativo equivalente ou superior aos plantios da África, Ásia e América Central.

Devido à qualidade de sua madeira, T. grandis apresenta grande valor comercial, podendo ser uma opção economicamente viável para as regiões que atendam suas condições edafoclimáticas. No entanto, o setor madeireiro brasileiro tem encontrado dificuldades na aquisição de madeiras nobres devido às pressões ambientais, pouca oferta de reflorestamento e de uma exploração seletiva de florestas nativas de forma sustentável (Pinto, 2007). Nesse sentido, torna-se necessário o conhecimento sobre suas exigências nutricionais para uma adequada avaliação do seu potencial produtivo (Favare et al., 2012).
Nesse caso, o solo será um dos fatores que influenciará no crescimento de $T$. grandis no campo (Gunaga et al., 2011).

Tonini et al. (2009) realizaram estudo estabelecendo uma comparação entre os atributos de um solo localizado em Iracema, Roraima, com as informações obtidas em literatura a respeito das demandas de $T$. grandis. Os autores constataram que, como a calagem e a adubação são fundamentais para o aumento da produtividade e o sucesso comercial da espécie, é necessário que se estabeleça, no estado de Roraima, ensaios de adubação nas suas diferentes condições edafoclimáticas, para que a fertilidade da área de plantio se torne adequada aos requerimentos da espécie.

Moya et al. (2013) recomendaram a adubação de plantio com $\mathrm{N}, \mathrm{K}$ e $\mathrm{Ca}$, pois estes são os nutrientes mais absorvidos por $T$. grandis. Além disso, a falta de $\mathrm{P}$ e de $\mathrm{B}$ pode limitar a produtividade dos plantios. Em contradição, Markewitz et al. (2012), estudando o solo e a resposta de $T$. grandis em decorrência da adubação fosfatada, não verificaram aumento no crescimento com a dosagem de $100 \mathrm{~kg} \mathrm{ha}^{-1}$ de P em 24 anos.

Para estabelecer as demandas nutricionais da espécie faz-se necessário conhecer o seu processo de adubação a nível de campo, bem como os custos desta prática. De acordo com a Federação de Agricultura e Pecuária do Estado de Mato Grosso FAMATO (2013), os custos com as atividades de plantio, que correspondem à sulcagem, adubação, plantio e replantio (10\% da área) são de $\mathrm{R} \$ 267,56 / \mathrm{ha}$. Sendo que a calagem e a gessagem em $100 \%$ da área contribuem com $\mathrm{R} \$ 12,36 /$ ha e a sulcagem e adubação com R \$ 230,00/ha. Portanto, são os maiores custos no momento do plantio. Nesse caso, apenas os custos com insumos ficam em torno de $\mathrm{R} \$$ 2.315,32/ha, reduzindo nos anos subsequentes. Porém, ao final de 20 anos, gera-se receita de R\$ 295.221/ha, com preço médio de $\mathrm{R} \$ 1.000,00 / \mathrm{m}^{3}$.

Portanto, além do crescimento em viveiro, outra necessidade é verificar como se dá o desenvolvimento da espécie em campo e, assim, estabelecer um programa de adubação, para reduzir gastos e aumentar o incremento da mesma. Diante disso, o presente experimento teve por objetivo avaliar a influência da adubação mineral NPK no crescimento e desenvolvimento das mudas de $T$. grandis no campo. 
VIEIRA, C.R.; WEBER, O.L. dos S.; SCARAMUZZA, J.F. Fertilização NPK no crescimento da teca.

\section{MATERIAL E MÉTODOS}

O experimento foi implantado, inicialmente, na casa de vegetação da Faculdade de Agronomia, Medicina Veterinária e Zootecnia - FAMEV, da Universidade Federal de Mato Grosso - UFMT, com mudas de $T$. grandis doadas pela KLM Florestal. As mudas foram produzidas em tubetes de $54 \mathrm{~cm}^{3}$ com substrato comercial Plantmax ${ }^{\circledR}$, composto por casca de pinus, fibra de coco; com a adição de vermiculita. Nessas condições, permaneceram até os 60 dias, quando atingiram porte para serem transplantadas.

Tabela 1. Análise química do solo.

Table 1. Soil chemical analysis.
Para este experimento com adubações NPK, utilizou-se o solo do tipo Latossolo vermelho distrófico de textura franco arenosa, tanto na fase de viveiro quanto na fase de campo. O solo foi coletado em área de vegetação nativa das condições de Cerrado, localizada no Instituto Federal de Mato Grosso, campus de São Vicente da Serra. Anteriormente às adições dos adubos, uma amostra desse solo foi previamente seca, peneirada em malha de $2 \mathrm{~mm}$ e caracterizada quimicamente seguindo os métodos descritos em Empresa Brasileira de Pesquisa Agropecuária - EMBRAPA(1997), conforme Tabela 1.

\begin{tabular}{|c|c|c|c|c|c|c|c|c|c|c|c|}
\hline $\mathrm{pH}$ & $\mathrm{H}+\mathrm{Al}$ & $\mathrm{Al}$ & $\mathrm{Ca}$ & $\mathrm{Mg}$ & $\mathrm{K}$ & $\mathrm{P}$ & SB & CTC & $\mathrm{t}$ & V & $\mathrm{m}$ \\
\hline $\mathrm{CaCl}_{2}$ & \multicolumn{4}{|c|}{$\mathrm{cmol}_{\mathrm{c}} \mathrm{dm}^{-3}$} & \multicolumn{2}{|c|}{$\mathrm{mg} \mathrm{dm}-^{3}$} & \multicolumn{3}{|c|}{$\mathrm{cmol}_{\mathrm{c}} \mathrm{dm}^{-3}$} & \multicolumn{2}{|c|}{$\%$} \\
\hline 4,39 & 4,22 & 1,03 & 1,0 & 0,50 & 13,56 & 13,9 & 1,53 & 5,75 & 2,56 & 26,5 & 40,2 \\
\hline
\end{tabular}

pH em $\mathrm{CaCl}_{2}$ - relação 1:2,5; $\mathrm{H}+\mathrm{Al}$ - em acetato de cálcio; $\mathrm{Al}, \mathrm{Ca}^{2}+$ e $\mathrm{Mg}^{2}+-$ em $\mathrm{KCl} 1 \mathrm{~N} ; \mathrm{P}$ e $\mathrm{K}$ - em Mehlich; $\mathrm{SB}$ - soma de bases; CTC - capacidade de troca de cátions a pH 7,0; t efetiva - CTC efetiva; V\% - saturação por bases, em \%; m\% - saturação por $\mathrm{Al}$, em \%.

$\mathrm{pH}$ in $\mathrm{CaCl}_{2}-1: 2.5$ ratio; $\mathrm{H}+\mathrm{Al}$ - in calcium acetate; $\mathrm{Al}, \mathrm{Ca}^{2}+$ and $\mathrm{Mg}^{2}+$ - in $\mathrm{KCl} 1 \mathrm{~N} ; \mathrm{P}$ and $\mathrm{K}$ - in Mehlich; $\mathrm{SB}$ - sum of bases; CTC - cation exchange capacity at $\mathrm{pH} 7.0$; $\mathrm{t}$ effective - effective CTC; V\% - base saturation, in $\%$; $\mathrm{m} \%$ - saturation per $\mathrm{Al}$, in $\%$.

Após a caracterização química do solo e anteriormente às adições de NPK, efetuou-se a calagem, buscando-se elevar a saturação por bases para $50 \%$. Para isso, foram consideradas as características do calcário utilizado, de PRNT 100\%, $30,08 \% \mathrm{CaO}$ e $21,1 \% \mathrm{MgO}$, necessitando-se o total de $1,53 \mathrm{t} \mathrm{ha}^{-1}$ do mesmo. O solo calcareado passou por período de incubação de 15 dias.

O cálculo da quantidade de calcário necessária para a elevação da saturação por bases foi realizado conforme a análise química do solo em condições naturais (Tabela 1), seguindo a equação de necessidade de calagem, proposta pela Embrapa:

$$
N C\left(_{\text {thd }}\right)=\left(V_{E}-V_{A}\right) T / 100
$$

Em que: $\mathrm{NC}=$ necessidade de calagem em toneladas por hectare; $\mathrm{V}_{\mathrm{E}}=$ saturação por bases desejada, em $\% ; \mathrm{V}_{\mathrm{A}}=$ saturação por bases atual, em \%; $\mathrm{T}=\mathrm{CTC}$ a pH 7,0.

Após o período de incubação do calcário, o solo foi dividido em diferentes recipientes, para as adições dos adubos e das doses testadas. A adubação se deu com diferentes níveis de NPK, utilizando-se como fonte de $\mathrm{N}$ a ureia, nas doses de
$0,40,80$ e $120 \mathrm{~kg} \mathrm{ha}^{-1}$; como fonte de $\mathrm{P}_{2} \mathrm{O}_{5}$, o superfosfato simples, nas doses de $0,50,100 \mathrm{e}$ $200 \mathrm{~kg} \mathrm{ha}^{-1}$ e como fonte de $\mathrm{K}_{2} \mathrm{O}$, o cloreto de potássio, nas doses de $0,50,100$ e $200 \mathrm{~kg} \mathrm{ha}^{-1}$. Posteriormente, o solo calcareado e adubado foi colocado em diferentes sacolas plásticas com tamanho de 50 x $40 \mathrm{~cm}$, considerando uma disposição em esquema fatorial de $4 \times 4 \times 4$, em blocos casualizados, com 12 repetições.

Após período 10 dias, que correspondeu à incubação do solo calcareado e adubado, as mudas atingiram $15 \mathrm{~cm}$ altura e foram transplantadas para as sacolas plásticas. Após o transplante, essas mudas passaram por período de adaptação de 15 dias para, em seguida, iniciar o acompanhamento do crescimento das mudas no viveiro.

As mudas foram mantidas até o final de 150 dias (a partir do acompanhamento do crescimento) nas condições de viveiro, em irrigação diária, em local com a intensidade luminosa semelhante, porém, sem controle da temperatura e/ou manutenção da adubação. Essas mudas foram medidas em altura, com fita métrica, a partir da superfície do substrato até a última folha totalmente formada, e em diâmetro, com paquímetro digital. 
VIEIRA, C.R.; WEBER, O.L. dos S.; SCARAMUZZA, J.F. Fertilização NPK no crescimento da teca.

As plantas obtidas dos 20 tratamentos que apresentaram as maiores médias para os crescimentos em altura e em diâmetro, conjuntamente, foram escolhidas para o plantio no campo (Tabela 2). Não sendo possível transplantar indivíduos representantes de todos os tratamentos devido à logística e ao tamanho da área de pesquisa.
A área de plantio das mudas de T. grandis foi disponibilizada pelo Instituto Federal de Mato Grosso, campus de São Vicente, utilizada anteriormente para pastagem (por 10 anos), localizada em ambiente de ocorrência de vegetação típica de Cerrado, em solo do tipo Latossolo Vermelho distrófico de textura franco arenosa, cujas condições químicas foram descritas na Tabela 1 .

Tabela 2. Tratamentos aplicados no plantio das mudas de Tectona grandis.

Table 2. Treatments applied to the planting of Tectona grandis seedlings.

\begin{tabular}{cccc}
\hline Tratamento & N-P-K & Tratamento & N-P-K \\
\hline 1 & $0-0-0$ & 11 & $40-200-100$ \\
2 & $40-0-100$ & 12 & $80-0-50$ \\
3 & $40-0-200$ & 13 & $80-50-50$ \\
4 & $40-50-0$ & 14 & $80-50-100$ \\
5 & $40-50-100$ & 15 & $80-100-100$ \\
6 & $40-100-50$ & 16 & $80-200-0$ \\
7 & $40-100-100$ & 17 & $120-50-0$ \\
8 & $40-100-200$ & 18 & $120-50-100$ \\
9 & $40-200-0$ & 19 & $120-100-50$ \\
10 & $40-200-50$ & 20 & $120-200-200$ \\
\hline
\end{tabular}

Para o plantio no campo, o solo foi gradeado e nivelado para melhoria de suas condições físicas e retirada de ervas daninhas. As covas foram abertas em espaçamento de 4 x $4 \mathrm{~m}$ com uma profundidade de $40 \mathrm{~cm}$, ao longo da área de plantio.

A calagem foi realizada diretamente na cova, utilizando-se calcário com as mesmas condições de PRNT e teores de $\mathrm{CaO}$ e $\mathrm{MgO}$ iguais aos da fase de crescimento em viveiro. Considerando que as condições de solo foram semelhantes, a quantidade de calcário utilizado nessa fase foi a mesma que para a fase de viveiro. Após a adição do calcário, este foi tampado com solo retirado da própria cova e, posteriormente, realizou-se a irrigação da mesma apenas para umedecer o produto e melhorar suas condições de reação.

Transcorridos sete dias da calagem, foram adicionados os adubos, também diretamente nas covas. Para isso, foram consideradas as mesmas fontes e doses empregadas na fase de viveiro e os tratamentos escolhidos para o transplante no campo (Tabela 2). A disposição final dos tratamentos foi a de blocos casualizados, com seis repetições.
Após 15 dias, as mudas foram plantadas nas covas calcareadas e adubadas. Nos primeiros 10 dias, houve irrigação diária, devido à baixa taxa de precipitação na região. Nos demais meses, as plantas foram mantidas nas condições naturais de campo, assim permanecendo por 12 meses. Durante esse período, as manutenções de plantio, como capinas, foram realizadas a cada 30 dias, sendo as capinas em área total e coroamento manual para cada planta.

Durante o período de crescimento das mudas no campo, efetuou-se a caracterização do crescimento com as medições de altura, com trena métrica, e de diâmetro, com paquímetro digital; aos 3, 6, 9 e 12 meses.

Após 12 meses, as plantas foram totalmente retiradas de cada cova e seccionadas em folhas, caule e raízes. O material foi seco em estufa de circulação forçada de ar a $65{ }^{\circ} \mathrm{C}$ até peso constante e pesado em balança semianalítica com precisão de 0,01 g. O material foliar seco foi moído em moinho tipo Wiley para posteriores análises de macro e de micronutrientes, conforme métodos de Malavolta et al. (1997). 
VIEIRA, C.R.; WEBER, O.L. dos S.; SCARAMUZZA, J.F. Fertilização NPK no crescimento da teca.

Para o processamento e análises dos dados foi utilizado o Assistat 7.6 beta, e a análise estatística foi realizada aplicando-se a técnica de análise de variância e comparação múltiplas de médias pelo método de Scott-Knott considerando significância de 5\%.

\section{RESULTADOS E DISCUSSÃO}

\subsection{Altura}

O crescimento em altura das plantas foi superior nos tratamentos com adubação (Tabela 3). Após as primeiras medições, aos três meses de crescimento, observou-se que as mudas do tratamento $16(80-200-0)$ foram as que obtiveram o maior crescimento em altura,
$58 \%$ superior ao das mudas no tratamento $1(0-0-0)$, indicando baixa exigência inicial pela adubação potássica para o incremento da altura. Resultado semelhante ao observado por Favare (2010) ao analisar o crescimento de $T$. grandis em doses crescentes de $\mathrm{K}$.

No período de três a seis meses de crescimento no campo, outros tratamentos também começaram a causar efeito no crescimento das mudas de $T$. grandis, com destaque para: 5 (40-50-100), 7 (40-100-100), 9 (40-200-0), 11 (40-200-100), 12 (80-0-50), 14 (80-50-100), 16 (80-200-0) e 19 (120-100-50). Demonstrando que a necessidade pela adubação potássica pode se dar após 90 dias do crescimento das mudas no campo. Em contrapartida, a efetiva necessidade por $\mathrm{N}$ e por $\mathrm{P}$ se dá desde o começo do estabelecimento de $T$. grandis no campo.

Tabela 3. Altura (em cm) das mudas de Tectona grandis submetidas a diferentes níveis de adubação NPK, durante 12 meses. Table 3. Height (in $\mathrm{cm}$ ) of Tectona grandis seedlings submitted to different levels of NPK fertilization, during 12 months.

\begin{tabular}{|c|c|c|c|c|}
\hline Tratamento (NPK) & Altura 3 meses & Altura 6 meses & Altura 9 meses & Altura 12 meses \\
\hline (1) $0-0-0$ & $27,67 \mathrm{e}$ & $30,00 \mathrm{~b}$ & $32,00 \mathrm{~d}$ & $32,00 \mathrm{~b}$ \\
\hline (2) $40-0-100$ & $33,33 \mathrm{~d}$ & $41,50 \mathrm{~b}$ & $52,17 \mathrm{c}$ & $53,50 \mathrm{~b}$ \\
\hline (3) $40-0-200$ & $34,50 \mathrm{~d}$ & $50,00 \mathrm{~b}$ & $54,50 \mathrm{c}$ & $68,50 \mathrm{a}$ \\
\hline (4) $40-50-0$ & $21,00 \mathrm{e}$ & $46,00 \mathrm{~b}$ & $46,17 \mathrm{c}$ & $48,17 \mathrm{~b}$ \\
\hline (5) $40-50-100$ & $35,67 \mathrm{~d}$ & $58,50 \mathrm{a}$ & $67,17 \mathrm{~b}$ & $74,33 \mathrm{a}$ \\
\hline (6) $40-100-50$ & $44,83 \mathrm{c}$ & $47,00 \mathrm{~b}$ & $47,33 \mathrm{c}$ & $48,83 \mathrm{~b}$ \\
\hline (7) $40-100-100$ & $26,83 \mathrm{e}$ & $62,33 \mathrm{a}$ & $62,50 \mathrm{~b}$ & $62,83 \mathrm{a}$ \\
\hline (8) $40-100-200$ & $44,83 \mathrm{c}$ & $50,33 \mathrm{~b}$ & $56,33 \mathrm{c}$ & $56,33 \mathrm{~b}$ \\
\hline (9) 40-200-0 & $38,17 \mathrm{~d}$ & 74,67 a & $76,83 \mathrm{a}$ & $78,17 \mathrm{a}$ \\
\hline (10) 40-200-50 & $40,50 \mathrm{c}$ & $47,83 \mathrm{~b}$ & $53,17 \mathrm{c}$ & $78,33 \mathrm{a}$ \\
\hline (11) 40-200-100 & $35,00 \mathrm{~d}$ & $72,00 \mathrm{a}$ & 74,17 a & 74,17 a \\
\hline (12) $80-0-50$ & $56,17 \mathrm{~b}$ & $65,67 \mathrm{a}$ & $77,17 \mathrm{a}$ & $79,67 \mathrm{a}$ \\
\hline (13) $80-50-50$ & $29,17 \mathrm{e}$ & $36,67 \mathrm{~b}$ & $55,17 \mathrm{c}$ & $55,17 \mathrm{~b}$ \\
\hline (14) 80-50-100 & $56,00 \mathrm{~b}$ & $58,17 \mathrm{a}$ & $64,67 \mathrm{~b}$ & $65,33 \mathrm{a}$ \\
\hline (15) $80-100-100$ & $41,17 \mathrm{c}$ & $45,33 \mathrm{~b}$ & $50,00 \mathrm{c}$ & $50,83 \mathrm{~b}$ \\
\hline (16) 80-200-0 & 65,83 a & $65,83 \mathrm{a}$ & $67,00 \mathrm{~b}$ & $75,17 \mathrm{a}$ \\
\hline (17) 120-50-0 & $40,67 \mathrm{c}$ & $51,50 \mathrm{~b}$ & $64,17 \mathrm{~b}$ & $66,67 \mathrm{a}$ \\
\hline (18) $120-50-100$ & $38,67 \mathrm{~d}$ & $46,83 \mathrm{~b}$ & $51,67 \mathrm{c}$ & $54,00 \mathrm{~b}$ \\
\hline (19) $120-100-50$ & $57,00 \mathrm{~b}$ & $57,00 \mathrm{a}$ & $60,17 \mathrm{~b}$ & $69,50 \mathrm{a}$ \\
\hline (20) $120-200-200$ & $45,83 \mathrm{c}$ & $52,00 \mathrm{~b}$ & $56,67 \mathrm{c}$ & $59,33 \mathrm{~b}$ \\
\hline $\mathrm{F}$ & $13,68 \mathrm{~ns}$ & $3,24 * *$ & $5,83 * *$ & $4,02 * *$ \\
\hline $\mathrm{CV}(\%)$ & 18,60 & 28,93 & 19,38 & 24,76 \\
\hline
\end{tabular}

Médias seguidas de mesma letra não diferem entre si pelo teste Scott-Knott a $5 \%$.

Averages followed by the same letter do not differ from each other by the Scott-Knott test at $5 \%$. 
VIEIRA, C.R.; WEBER, O.L. dos S.; SCARAMUZZA, J.F. Fertilização NPK no crescimento da teca.

Aos 12 meses, os tratamentos $3(40-0-200)$, 5 (40-50-100), 7 (40-100-100), 9 (40-200-0), 10 (40-200-50), 11 (40-200-100), $12(80-0-50)$, 14 (80-50-100), 16 (80-200-0), 17 (120-50-0) e 19 (120-100-50) foram os que se destacaram. No entanto, os maiores incrementos em altura, durante os 12 meses, foram observados nos tratamentos 4 (40-50-0), 7 (40-100-100) e 11 (40-200-100), que atingiram médias superiores em $79,6 \%, 83,8 \%$ e $79,5 \%$, respectivamente, ao tratamento $1(0-0-0)$.

Favare (2010) também verificou influência do $\mathrm{N}$ e do $\mathrm{P}$ no crescimento em altura das mudas de $T$. grandis, recomendando as doses de $107 \mathrm{mg} \mathrm{kg}^{-1}$ de $\mathrm{N}$ e $200 \mathrm{mg} \mathrm{kg}^{-1}$ de $\mathrm{P}$, o que indica a importância dos nutrientes no desenvolvimento das plantas, mesmo após 360 dias do plantio.

Após 12 meses de estabelecimento, o crescimento médio de $T$. grandis submetida a adubações foi de $72 \mathrm{~cm}$ em 12 meses, condizente com o observado por Tonini et al. (2009) ao estabelecer curvas de crescimento em altura dominante para plantios de T. grandis em Roraima, Mato Grosso, Acre e na Costa Rica.

Portanto, em geral, as mudas de $T$. grandis submetidas às adubações com $40 \mathrm{~kg} \mathrm{ha}^{-1}$ de $\mathrm{N}$, $200 \mathrm{~kg} \mathrm{ha}^{-1}$ de $\mathrm{P}_{2} \mathrm{O}_{5}$ e $100 \mathrm{~kg} \mathrm{ha}^{-1}$ de $\mathrm{K}_{2} \mathrm{O}$ obtiveram maior crescimento em altura ao longo de 12 meses de desenvolvimento no campo, correspondendo ao tratamento 11 .

\subsection{Diâmetro}

$\mathrm{O}$ crescimento em diâmetro foi influenciado pela adubação NPK (Tabela 3), destacando-se, inicialmente, no tratamento 19 (120-100-50), com média $56 \%$ superior ao tratamento 1 (0-0-0), reforçando a necessidade de $\mathrm{N}, \mathrm{P}$ e $\mathrm{K}$ na adubação de plantio, com menor influência do $\mathrm{K}$ nessa característica durante os primeiros três meses de crescimento. Porém, diferentemente do que ocorreu para o crescimento em altura, a adubação potássica foi importante para o crescimento em diâmetro desde os primeiros meses de desenvolvimento de $T$. grandis, observando-se resultado na dose de $50 \mathrm{~kg} \mathrm{ha}^{-1}$.

No período de três a seis meses, observou-se o maior crescimento nas mudas dos tratamentos 11 (40-200-100), $12 \quad(80-0-50)$, 16 (80-200-0), 17 (120-50-0), 18 (80-200-100), $19(120-100-50)$ e 20 (120-200-200), com médias que foram $23 \%, 32 \%, 26 \%, 24 \%, 31 \%, 43 \%$ e $36 \%$, respectivamente, superiores ao tratamento 1 (0-0-0). O que pode ter ocorrido é que, nessa fase os adubos estavam na máxima liberação dos nutrientes, o que favoreceu sua disponibilidade e absorção pelas plantas, contribuindo para o crescimento da mesmas.

Tabela 4. Diâmetro (em mm) das mudas de Tectona grandis submetidas a diferentes níveis de adubação NPK, durante 12 meses.

Table 4. Diameter (in $\mathrm{mm}$ ) of Tectona grandis seedlings submitted to different levels of NPK fertilization, during 12 months.

\begin{tabular}{ccccc}
\hline Tratamento (NPK) & Diâmetro 3 meses & Diâmetro 6 meses & Diâmetro 9 meses & Diâmetro 12 meses \\
\hline (1) $0-0-0$ & $7,68 \mathrm{~d}$ & $12,62 \mathrm{~b}$ & $12,65 \mathrm{c}$ & $13,27 \mathrm{c}$ \\
$(2) 40-0-100$ & $11,57 \mathrm{c}$ & $12,28 \mathrm{~b}$ & $15,22 \mathrm{c}$ & $18,53 \mathrm{~b}$ \\
$(3) 40-0-200$ & $13,00 \mathrm{~b}$ & $11,42 \mathrm{~b}$ & $12,23 \mathrm{c}$ & $13,67 \mathrm{c}$ \\
$(4) 40-50-0$ & $11,28 \mathrm{c}$ & $12,60 \mathrm{~b}$ & $13,72 \mathrm{c}$ & $13,75 \mathrm{c}$ \\
$(5) 40-50-100$ & $12,68 \mathrm{~b}$ & $14,40 \mathrm{~b}$ & $16,60 \mathrm{~b}$ & $18,03 \mathrm{~b}$ \\
(6) $40-100-50$ & $13,08 \mathrm{~b}$ & $15,05 \mathrm{~b}$ & $17,50 \mathrm{~b}$ & $18,58 \mathrm{~b}$ \\
(7) $40-100-100$ & $11,00 \mathrm{c}$ & $11,47 \mathrm{~b}$ & $11,65 \mathrm{c}$ & $13,77 \mathrm{c}$ \\
(8) $40-100-200$ & $11,33 \mathrm{c}$ & $12,33 \mathrm{~b}$ & $12,92 \mathrm{c}$ & $14,28 \mathrm{c}$
\end{tabular}

continua to be continued 
VIEIRA, C.R.; WEBER, O.L. dos S.; SCARAMUZZA, J.F. Fertilização NPK no crescimento da teca.

continuação - Tabela 4

continuation - Table 4

\begin{tabular}{ccccc}
\hline Tratamento (NPK) & Diâmetro 3 meses & Diâmetro 6 meses & Diâmetro 9 meses & Diâmetro 12 meses \\
\hline (9) $40-200-0$ & $13,12 \mathrm{~b}$ & $15,22 \mathrm{~b}$ & $20,68 \mathrm{a}$ & $21,08 \mathrm{~b}$ \\
$(10) 40-200-50$ & $10,97 \mathrm{c}$ & $14,03 \mathrm{~b}$ & $15,17 \mathrm{c}$ & $16,87 \mathrm{c}$ \\
$(11) 40-200-100$ & $14,00 \mathrm{~b}$ & $16,47 \mathrm{a}$ & $18,22 \mathrm{~b}$ & $18,43 \mathrm{~b}$ \\
$(12) 80-0-50$ & $11,30 \mathrm{c}$ & $18,73 \mathrm{a}$ & $22,80 \mathrm{a}$ & $22,20 \mathrm{a}$ \\
$(13) 80-50-50$ & $8,85 \mathrm{~d}$ & $12,78 \mathrm{~b}$ & $12,90 \mathrm{c}$ & $13,70 \mathrm{c}$ \\
$(14) 80-50-100$ & $11,63 \mathrm{c}$ & $15,03 \mathrm{~b}$ & $18,10 \mathrm{~b}$ & $18,53 \mathrm{~b}$ \\
$(15) 80-100-100$ & $9,88 \mathrm{~d}$ & $11,98 \mathrm{~b}$ & $12,05 \mathrm{c}$ & $12,08 \mathrm{c}$ \\
$(16) 80-200-0$ & $13,28 \mathrm{~b}$ & $17,13 \mathrm{a}$ & $19,37 \mathrm{~b}$ & $19,43 \mathrm{~b}$ \\
$(17) 120-50-0$ & $13,68 \mathrm{~b}$ & $16,58 \mathrm{a}$ & $19,43 \mathrm{~b}$ & $19,22 \mathrm{~b}$ \\
$(18) 120-50-100$ & $13,50 \mathrm{~b}$ & $18,35 \mathrm{a}$ & $17,15 \mathrm{~b}$ & $18,20 \mathrm{~b}$ \\
$(19) 120-100-50$ & $17,40 \mathrm{a}$ & $22,37 \mathrm{a}$ & $24,48 \mathrm{a}$ & $25,65 \mathrm{a}$ \\
$(20) 120-200-200$ & $14,80 \mathrm{~b}$ & $19,72 \mathrm{a}$ & $20,68 \mathrm{a}$ & $25,63 \mathrm{a}$ \\
\hline $\mathrm{F}$ & $4,75^{* *}$ & $4,97 * *$ & $4,98^{* *}$ & $3,63 * *$ \\
\hline CV(\%) & 19,79 & 22,34 & 25,04 & 28,46 \\
\hline
\end{tabular}

Médias seguidas de mesma letra não diferem entre si pelo teste Scott-Knott a $5 \%$.

Averages followed by the same letter do not differ from each other by the Scott-Knott test at $5 \%$.

No período de seis a nove meses, o crescimento em diâmetro foi superior nos tratamentos 9 (40-200-0), 12 (80-0-50), 19 (120-100-50) e 20 (120-200-200), com médias que foram $39 \%, 44 \%, 48 \%$ e $39 \%$, respectivamente, superiores ao tratamento $1(0-0-0)$, enquanto no período de nove a doze meses, o crescimento em diâmetro continuou sendo destaque nos tratamentos 12 (80-0-50), 19 (120-100-50) e 20 (120-200-200), que foram $40 \%, 48 \%$ e $48 \%$, respectivamente, superiores ao tratamento 1 (0-0-0). Esses resultados indicam que as exigências das plantas por $\mathrm{N}$ aumentam com o tempo, dependendo da característica morfológica analisada.

Nesse caso, os maiores incrementos, durante os 12 meses, foram observados nas mudas submetidas ao tratamento 12 (80-0-50), em que, diferentemente do que ocorreu no crescimento em altura, o K foi importante para a formação do caule das plantas desde os primeiros meses de plantio, o que é interessante, porque esse crescimento dará origem à madeira, enquanto o requerimento em $\mathrm{P}$ foi menor, provavelmente, porque o elemento pode ser demandado em maiores concentrações na parte foliar da planta, na produção de energia.
Portanto, em geral, os níveis de adubação NPK que promoveram o maior crescimento em diâmetro foram $120 \mathrm{~kg} \mathrm{ha}^{-1}$ de N, $200 \mathrm{~kg} \mathrm{ha}^{-1}$ de $\mathrm{P}_{2} \mathrm{O}_{5}$ e $50 \mathrm{~kg} \mathrm{ha}^{-1}$ de $\mathrm{K}_{2} \mathrm{O}$. No entanto, o tratamento com os três níveis combinados não foi escolhido para o transplantio ao campo.

\subsection{Biomassa Seca}

Ao avaliar a produção de biomassa seca, ao final de 12 meses, verificou-se que esta também foi influenciada pela adubação NPK (Tabela 5).

Segundo Cruz et al. (2004), a produção de biomassa foliar é interessante para o melhor desenvolvimento das mudas, pois representa maior capacidade fotossintética e maior vigor. Nesse caso, o tratamento 12 (80-0-50) foi o que proporcionou maior produção de biomassa foliar. Portanto, assim como ocorreu nas medições dos demais parâmetros, o $\mathrm{N}$ foi o mais importante. No entanto, esses resultados também estão relacionados com a mobilidade do elemento e suas funções como a divisão celular e a fotossíntese, que influenciam diretamente na produção de biomassa foliar. 
VIEIRA, C.R.; WEBER, O.L. dos S.; SCARAMUZZA, J.F. Fertilização NPK no crescimento da teca.

Tabela 5. Biomassa seca das folhas, caule a raiz (em g) das mudas de Tectona grandis submetidas a diferentes níveis de adubação NPK, após 12 meses.

Table 5. Dry biomass of leaves, stem and roots (in g) of Tectona grandis seedlings submitted to different levels of NPK fertilization, after 12 months.

\begin{tabular}{cccc}
\hline Tratamento (NPK) & Biomassa folha & Biomassa caule & Biomassa raiz \\
\hline (1) $0-0-0$ & $15,17 \mathrm{~d}$ & $8,07 \mathrm{c}$ & $34,79 \mathrm{~d}$ \\
$(2) 40-0-100$ & $13,08 \mathrm{e}$ & $9,37 \mathrm{c}$ & $32,06 \mathrm{~d}$ \\
(3) $40-0-200$ & $16,96 \mathrm{~d}$ & $7,98 \mathrm{c}$ & $32,06 \mathrm{~d}$ \\
(4) $40-50-0$ & $10,13 \mathrm{e}$ & $8,34 \mathrm{c}$ & $28,69 \mathrm{e}$ \\
(5) $40-50-100$ & $20,69 \mathrm{~d}$ & $12,09 \mathrm{c}$ & $42,94 \mathrm{c}$ \\
(6) $40-100-50$ & $32,20 \mathrm{~b}$ & $29,76 \mathrm{~b}$ & $47,39 \mathrm{~b}$ \\
(7) $40-100-100$ & $6,79 \mathrm{e}$ & $7,99 \mathrm{c}$ & $16,43 \mathrm{f}$ \\
(8) $40-100-200$ & $15,99 \mathrm{~d}$ & $11,39 \mathrm{c}$ & $25,05 \mathrm{e}$ \\
(9) $40-200-0$ & $19,83 \mathrm{~d}$ & $18,83 \mathrm{c}$ & $37,98 \mathrm{~d}$ \\
(10) $40-200-50$ & $19,56 \mathrm{~d}$ & $16,22 \mathrm{c}$ & $38,53 \mathrm{~d}$ \\
(11) $40-200-100$ & $30,22 \mathrm{~b}$ & $41,54 \mathrm{a}$ & $51,45 \mathrm{~b}$ \\
(12) $80-0-50$ & $46,12 \mathrm{a}$ & $43,62 \mathrm{a}$ & $41,38 \mathrm{c}$ \\
(13) $80-50-50$ & $12,10 \mathrm{e}$ & $8,52 \mathrm{c}$ & $26,91 \mathrm{e}$ \\
(14) $80-50-100$ & $27,21 \mathrm{c}$ & $24,51 \mathrm{~b}$ & $50,31 \mathrm{~b}$ \\
(15) $80-100-100$ & $8,61 \mathrm{e}$ & $7,18 \mathrm{c}$ & $25,03 \mathrm{e}$ \\
(16) $80-200-0$ & $12,07 \mathrm{e}$ & $17,59 \mathrm{c}$ & $40,92 \mathrm{c}$ \\
(17) $120-50-0$ & $10,80 \mathrm{e}$ & $24,48 \mathrm{~b}$ & $30,57 \mathrm{c}$ \\
(18) $120-50-100$ & $27,66 \mathrm{c}$ & $26,86 \mathrm{~b}$ & $35,74 \mathrm{c}$ \\
(19) $120-100-50$ & $24,57 \mathrm{c}$ & $35,24 \mathrm{a}$ & $69,54 \mathrm{a}$ \\
(20) $120-200-200$ & $30,13 \mathrm{~b}$ & $32,87 \mathrm{~b}$ & $43,23 \mathrm{c}$ \\
\hline $\mathrm{F}$ & $30,79 * *$ & $9,72 * *$ & $25,94 * *$ \\
\hline $\mathrm{CV}(\%)$ & 21,95 & 48,02 & 15,08 \\
\hline
\end{tabular}

Médias seguidas de mesma letra não diferem entre si pelo teste Scott-Knott a 5\%.

Averages followed by the same letter do not differ from each other by the Scott-Knott test at $5 \%$.

De acordo com Marschner (1995) e Malavolta et al. (1997), o $\mathrm{N}$ é exigido em grandes quantidades no início do crescimento das plantas. Devido às suas funções na estrutura de aminoácidos, proteínas, bases nitrogenadas, ácidos nucléicos, enzimas, pigmentos e produtos secundários, participação na absorção iônica, fotossíntese, respiração, multiplicação e diferenciação celular; em caso de restrições, haverá redução de crescimento.

Com relação à baixa necessidade por $\mathrm{P}$ até os 12 meses de crescimento, Faria (1993) e Lima (1995) ressaltaram que uma elevada resposta a menores doses de $\mathrm{P}$ indica maior viabilidade do uso de espécies com baixa demanda por P na recuperação de áreas onde os solos apresentem restrições quanto à disponibilidade do nutriente.
Essas características nutricionais, juntamente com as outras características edáficas, ajudam a entender a adaptabilidade de $T$. grandis e suas altas produções nos solos do Cerrado mato-grossense.

Nicoloso et al. (2001) observaram que, dentre os vários parâmetros de crescimento avaliados em Apuleia leiocarpa, o número de folhas por planta foi o mais influenciado pelos tratamentos de adubação, com resposta às adubações isoladas de $\mathrm{N}, \mathrm{P}$ e $\mathrm{K}$, bem como à interação das adubações de NP e NK, fato que esteve fortemente correlacionado à produção de biomassa das folhas e, consequentemente, ao total da planta, o que explica a influência das adubações na produção de biomassa das mudas de $T$. grandis. 
VIEIRA, C.R.; WEBER, O.L. dos S.; SCARAMUZZA, J.F. Fertilização NPK no crescimento da teca.

Com relação à biomassa seca do caule, os tratamentos 11 (40-200-100), $12(80-0-50)$ e 19 (120-100-50) apresentaram as maiores médias, relacionando-se com o crescimento em diâmetro nesses tratamentos e influência do $\mathrm{K}$ nessa característica.

Para a biomassa seca da raiz, a maior média foi observada no tratamento 19 (120-100-50). Portanto, a presença de P influenciou na biomassa da parte radicular das plantas de $T$. grandis, devido às funções deste elemento no processo de produção energética para a divisão e diferenciação celular de partes novas das plantas. Esses resultados são importantes, segundo Gonçalves et al. (1992), porque espécies dos grupos ecológicos iniciais, que possuem sistema radicular mais desenvolvido e raízes finas em maior quantidade, apresentam as maiores taxas de crescimento.

Com relação à produção de biomassa, em geral, os tratamentos 12 (80-0-50) e 19 (120-100-50) foram os que propiciaram os maiores incrementos em mudas de $T$. grandis, observando-se uma necessidade de $\mathrm{K}$ semelhante em todas as partes das plantas, diferentemente do que ocorreu para $\mathrm{N}$ e para $\mathrm{P}$.

Mutanal et al. (2000) recomendaram a adubação com $100 \mathrm{~kg} \mathrm{ha}^{-1}$ de $\mathrm{N}, 50 \mathrm{~kg} \mathrm{ha}^{-1}$ de $\mathrm{P}_{2} \mathrm{O}_{5}$ e $100 \mathrm{~kg} \mathrm{ha}^{-1}$ de $\mathrm{K}_{2} \mathrm{O}$ no primeiro ano de plantio de $T$. grandis, enquanto Prasad et al. (1986) recomendaram a aplicação de 300 e $150 \mathrm{~kg} \mathrm{ha}^{-1}$ de $\mathrm{N}$ e de $\mathrm{P}_{2} \mathrm{O}_{5}$ anualmente, por cinco anos.
Comparando-se os resultados obtidos nas medições de crescimento em altura e em diâmetro das plantas de $T$. grandis com os de Mutanal et al. (2000), observou-se semelhança nas recomendações de $\mathrm{N}$, discordando-se das verificadas para $\mathrm{P}_{2} \mathrm{O}_{5}$ e de $\mathrm{K}_{2} \mathrm{O}$, enquanto nos dados relacionados à produção de biomassa, as recomendações de $\mathrm{N}$ e de $\mathrm{P}_{2} \mathrm{O}_{5}$ foram semelhantes para $\mathrm{N}$ e para $\mathrm{P}$, o que pode estar relacionado à mudança de condições ambientais entre os experimentos.

Portanto, o crescimento das mudas de T. grandis em campo foi positivamente influenciado pela adubação NPK. Observando-se, em geral, o menor crescimento no tratamento sem adubação (testemunha). Resultados semelhantes aos observados por Mutanal et al. (2000), Gunaga et al. (2011) e Moya et al. (2013) ao testar a adubação em plantios de $T$. grandis.

\subsection{Nutrição das mudas de Tectona grandis}

Além das características morfológicas, há que se observar se as concentrações dos nutrientes estão de acordo com as necessidades da planta e se estas, por sua vez, foram influenciadas pelas doses aplicadas. Sendo assim, foram determinadas as concentrações de macronutrientes e apresentadas na Tabela 6. Ressaltando-se que não se observou diferença nas concentrações de $\mathrm{N}$ e de $\mathrm{K}$ nas mudas.

Tabela 6. Concentrações de macronutrientes, em $\mathrm{g} \mathrm{kg}^{-1}$, nas folhas de Tectona grandis submetidas a diferentes níveis de adubação NPK, após 12 meses.

Table 6. Macronutrient concentrations, in $\mathrm{g} \mathrm{kg}^{-1}$, in leaves of Tectona grandis submitted to different levels of NPK fertilization, after 12 months.

\begin{tabular}{ccccccc}
\hline Tratamento (NPK) & $\mathrm{N}$ & $\mathrm{P}$ & $\mathrm{K}$ & $\mathrm{Ca}$ & $\mathrm{Mg}$ & $\mathrm{S}$ \\
\hline (1) $0-0-0$ & $8,67 \mathrm{a}$ & $2,77 \mathrm{a}$ & $9,82 \mathrm{a}$ & $1,36 \mathrm{~b}$ & $1,29 \mathrm{~b}$ & $0,19 \mathrm{e}$ \\
(2) $40-0-100$ & $7,47 \mathrm{a}$ & $3,82 \mathrm{a}$ & $8,97 \mathrm{a}$ & $1,20 \mathrm{~b}$ & $1,63 \mathrm{~b}$ & $0,34 \mathrm{e}$ \\
(3) $40-0-200$ & $8,60 \mathrm{a}$ & $3,22 \mathrm{a}$ & $12,26 \mathrm{a}$ & $1,12 \mathrm{~b}$ & $2,25 \mathrm{~b}$ & $0,83 \mathrm{~d}$ \\
(4) $40-50-0$ & $7,67 \mathrm{a}$ & $3,10 \mathrm{a}$ & $7,87 \mathrm{a}$ & $1,28 \mathrm{~b}$ & $2,49 \mathrm{~b}$ & $1,16 \mathrm{~b}$ \\
(5) $40-50-100$ & $9,23 \mathrm{a}$ & $3,10 \mathrm{a}$ & $8,97 \mathrm{a}$ & $1,84 \mathrm{a}$ & $1,92 \mathrm{~b}$ & $0,92 \mathrm{c}$
\end{tabular}

continua to be continued 
VIEIRA, C.R.; WEBER, O.L. dos S.; SCARAMUZZA, J.F. Fertilização NPK no crescimento da teca.

continuação - Tabela 6

continuation - Table 6

\begin{tabular}{ccccccc}
\hline Tratamento (NPK) & $\mathrm{N}$ & $\mathrm{P}$ & $\mathrm{K}$ & $\mathrm{Ca}$ & $\mathrm{Mg}$ & $\mathrm{S}$ \\
\hline (6) $40-100-50$ & $10,30 \mathrm{a}$ & $3,72 \mathrm{a}$ & $8,38 \mathrm{a}$ & $1,36 \mathrm{~b}$ & $2,97 \mathrm{a}$ & $1,24 \mathrm{~b}$ \\
$(7) 40-100-100$ & $9,27 \mathrm{a}$ & $2,97 \mathrm{a}$ & $7,62 \mathrm{a}$ & $1,28 \mathrm{~b}$ & $2,73 \mathrm{a}$ & $0,99 \mathrm{c}$ \\
$(8) 40-100-200$ & $7,37 \mathrm{a}$ & $3,50 \mathrm{a}$ & $4,33 \mathrm{a}$ & $1,76 \mathrm{a}$ & $2,97 \mathrm{a}$ & $0,62 \mathrm{~d}$ \\
(9) $40-200-0$ & $7,37 \mathrm{a}$ & $3,45 \mathrm{a}$ & $6,44 \mathrm{a}$ & $0,96 \mathrm{~b}$ & $3,07 \mathrm{a}$ & $0,95 \mathrm{c}$ \\
$(10) 40-200-50$ & $8,50 \mathrm{a}$ & $3,40 \mathrm{a}$ & $9,06 \mathrm{a}$ & $1,44 \mathrm{a}$ & $2,11 \mathrm{~b}$ & $0,58 \mathrm{~d}$ \\
$(11) 40-200-100$ & $9,83 \mathrm{a}$ & $0,72 \mathrm{~b}$ & $6,52 \mathrm{a}$ & $1,28 \mathrm{~b}$ & $2,59 \mathrm{~b}$ & $2,08 \mathrm{a}$ \\
$(12) 80-0-50$ & $6,73 \mathrm{a}$ & $3,45 \mathrm{a}$ & $7,96 \mathrm{a}$ & $1,28 \mathrm{~b}$ & $3,31 \mathrm{a}$ & $0,61 \mathrm{~d}$ \\
$(13) 80-50-50$ & $10,10 \mathrm{a}$ & $1,32 \mathrm{~b}$ & $9,40 \mathrm{a}$ & $1,60 \mathrm{a}$ & $2,21 \mathrm{~b}$ & $1,39 \mathrm{~b}$ \\
$(14) 80-50-100$ & $7,20 \mathrm{a}$ & $1,60 \mathrm{~b}$ & $8,81 \mathrm{a}$ & $1,52 \mathrm{a}$ & $2,78 \mathrm{a}$ & $1,35 \mathrm{~b}$ \\
$(15) 80-100-100$ & $6,90 \mathrm{a}$ & $3,07 \mathrm{a}$ & $9,14 \mathrm{a}$ & $1,44 \mathrm{a}$ & $2,59 \mathrm{~b}$ & $0,36 \mathrm{e}$ \\
$(16) 80-200-0$ & $8,97 \mathrm{a}$ & $3,12 \mathrm{a}$ & $6,61 \mathrm{a}$ & $1,52 \mathrm{a}$ & $3,21 \mathrm{a}$ & $0,71 \mathrm{~d}$ \\
(17) $120-50-0$ & $10,60 \mathrm{a}$ & $3,67 \mathrm{a}$ & $6,27 \mathrm{a}$ & $1,12 \mathrm{~b}$ & $3,60 \mathrm{a}$ & $0,94 \mathrm{c}$ \\
$(18) 120-50-100$ & $7,27 \mathrm{a}$ & $3,20 \mathrm{a}$ & $9,23 \mathrm{a}$ & $0,96 \mathrm{~b}$ & $3,55 \mathrm{a}$ & $1,09 \mathrm{c}$ \\
$(19) 120-100-50$ & $8,87 \mathrm{a}$ & $2,35 \mathrm{a}$ & $9,23 \mathrm{a}$ & $1,28 \mathrm{~b}$ & $2,64 \mathrm{~b}$ & $1,23 \mathrm{~b}$ \\
$(20) 120-200-200$ & $9,43 \mathrm{a}$ & $3,37 \mathrm{a}$ & $11,93 \mathrm{a}$ & $1,44 \mathrm{a}$ & $3,84 \mathrm{a}$ & $0,93 \mathrm{c}$ \\
\hline $\mathrm{F}$ & $1,27 \mathrm{~ns}$ & $5,08 * *$ & $2,40 \mathrm{~ns}$ & $3,56 * *$ & $4,12 * *$ & $13,09 * *$ \\
\hline CV(\%) & 21,74 & 24,99 & 24,73 & 18,08 & 24,25 & 25,83 \\
\hline
\end{tabular}

Médias seguidas de mesma letra não diferem entre si pelo teste Scott-Knott a $5 \%$.

Averages followed by the same letter do not differ from each other by the Scott-Knott test at $5 \%$.

A adubação de plantio com NPK proporcionou concentrações de N (Tabela 6), aos 12 meses, inferiores às observados por Favare (2010), aos 240 dias, ao aplicar doses crescentes de $\mathrm{N}$ em plantas de Tectona grandis. Isso pode indicar a necessidade de adubação de manutenção antes do período de 12 meses, já que podem ter ocorrido, além da absorção pela planta, perdas do nutriente. Porém, essas concentrações não parecem ter influenciado negativamente $\mathrm{o}$ crescimento inicial das plantas no campo. Segundo Behling (2009), espécies florestais, principalmente as de rápido crescimento, apresentam maior taxa de absorção e retenção de nutrientes na fase juvenil, fato que pode justificar a redução nas concentrações de $\mathrm{N}$ e as médias obtidas aos 12 meses, o que foi comprovado por Moya et al. (2013), ao observarem redução em T. grandis localizadas na Costa Rica e no Panamá.

$\mathrm{O} \mathrm{P}$ é um dos nutrientes que tem merecido maior preocupação em razão de sua baixa disponibilidade natural nos solos mais intemperizados (Ribeiro et al., 2006), pois, entre as funções que exerce no metabolismo da planta, tem a capacidade de melhorar $o$ desenvolvimento do sistema radicular das plantas (Gonçalves et al., 2000). Além disso, a quantidade de $\mathrm{P}$ irá influenciar na contração radial e tangencial da madeira (Moya et al., 2010), por isso, concentrações adequadas do elemento, desde a fase inicial de crescimento no campo será primordial.

Com relação ao P (Tabela 6), as plantas nos tratamentos 11 (40-200-100), 13 (80-50-50) e 14 (80-50-100) foram as que apresentaram as menores concentrações na parte foliar. Esses resultados ajudam a explicar o crescimento inicial de $T$. grandis no campo, com as maiores exigências em $\mathrm{P}$ na fase dos três primeiros meses, verificando-se, posteriormente, uma redução. Por isso, ao final de 12 meses, observaram-se essas menores concentrações, principalmente no tratamento 11, que propiciou os maiores crescimentos em altura. A necessidade de $\mathrm{P}$ em plantios de $T$. grandis também já foi constatada por Ribeiro et al. (2006), Tonini et al. (2009), Favare (2013) e Moya et al. (2013). 
VIEIRA, C.R.; WEBER, O.L. dos S.; SCARAMUZZA, J.F. Fertilização NPK no crescimento da teca.

Não houve diferença significativa nas concentrações de K (Tabela 6), no entanto, os tratamentos 3 (40-0-200) e 20 (120-200-200) foram os únicos que proporcionaram concentrações acima de $10 \mathrm{~g} \mathrm{~kg}^{-1}$ de $\mathrm{K}$ nas folhas de $T$. grandis após 12 meses. Uma adequada nutrição com $\mathrm{K}$ pode propiciar melhores condições de desenvolvimento das plantas e, consequentemente, maior acúmulo de biomassa seca nas mesmas (Favare, 2010). O que pode explicar o crescimento de $T$. grandis, principalmente, nas doses de $50 \mathrm{~kg} \mathrm{ha}^{-1}$ de $\mathrm{K}_{2} \mathrm{O}$ para o crescimento em diâmetro e de $100 \mathrm{~kg} \mathrm{ha}^{-1}$ de $\mathrm{K}_{2} \mathrm{O}$ para o crescimento em altura.

As maiores concentrações de $\mathrm{Ca}$ (Tabela 6) foram observadas nos tratamentos 5 (40-50-100), 8 (40-100-200), 10 (40-200-50), 13 (80-50-50), 14 (80-50-100), 15 (80-100-100), 16 (80-200-0) e 20 (120-200-200). Resultados que também estão relacionados à utilização do superfosfato simples para a adubação NPK, já que o mesmo também possui $\mathrm{Ca}$ na sua composição e que pode ser observado diante dos tratamentos que proporcionaram as maiores concentrações de $\mathrm{Ca}$ até o final de 12 meses. Porém, as doses em que se observaram os maiores crescimentos não estão entre as que apresentaram as maiores concentrações de $\mathrm{Ca}$ ao final dos 12 meses, demonstrando os maiores requerimentos em períodos anteriores, portanto, durante o seu crescimento inicial. Mutanal et al. (2000), Ribeiro et al. (2006), Tonini et al. (2009), Gunaga et al. (2011) e Moya et al. (2013) comprovaram, anteriormente, as exigências de $T$. grandis em Ca.

Quanto ao Mg, as maiores concentrações (Tabela 6) foram observadas nos tratamentos 6 (40-100-50), 7 (40-100-100), 8 (40-100-200), 9 (40-200-0), 12 (80-0-50), 14 (80-50-100), 16 (80-200-0), 17 (120-50-0), 18 (120-50-100) e 20 (120-200-200). Prado e Vidal (2008) sugerem que a elevação dos teores de alguns nutrientes ocorre pelo efeito de concentração, pois o menor crescimento pode ter concentrado os nutrientes no tecido vegetal; isto pode ter acontecido nos tratamentos $6,8,18$ e 20, pois apresentaram os menores crescimentos em altura.
O tratamento 11 (40-200-100) propiciou as maiores concentrações de S (Tabela 6), ao final de 12 meses, provavelmente porque o superfosfato simples, que foi a fonte de $\mathrm{P}$ utilizada no experimento, também é fonte de $\mathrm{S}$, o que pode ter contribuído para maior crescimento em altura nesse tratamento, porém, indica uma demanda maior em $\mathrm{S}$ após os 12 meses.

Em geral, verificou-se que as doses que proporcionaram condições nutricionais ideais para T. grandis foram $40 \mathrm{~kg} \mathrm{ha}^{-1}$ de $\mathrm{N}, 50 \mathrm{~kg} \mathrm{ha}{ }^{-1}$ de $\mathrm{P}_{2} \mathrm{O}_{5}$ e 50 ou $100 \mathrm{~kg} \mathrm{ha}^{-1}$ de $\mathrm{K}_{2} \mathrm{O}$, contribuindo para o seu crescimento inicial.

Figueiredo e Sá (2005) estimaram, para uma adubação de plantio para $T$. grandis, com $15 \mathrm{~kg} \mathrm{ha}^{-1}$ de $\mathrm{N}, 75 \mathrm{~kg} \mathrm{ha}^{-1}$ de $\mathrm{P}_{2} \mathrm{O}_{5}$ e $15 \mathrm{~kg} \mathrm{ha}^{-1} \mathrm{~K}_{2} \mathrm{O}$, os custos para adubação em: $\mathrm{R} \$ 271,66$ em plantios com densidade de 1.111 mudas (espaçamento $3 \mathrm{~m} \times 3 \mathrm{~m}$ ); R $\$ 407,49$ em plantios com densidade de 1.667 mudas (espaçamento de $3 \mathrm{~m} \mathrm{x} 2 \mathrm{~m}$ ) e R\$ 489,95 em plantios com densidade de 2.000 mudas (espaçamento de $2 \mathrm{~m} \times 2,5 \mathrm{~m}$ ). Sendo os maiores custos com o adubo fosfatado: $\mathrm{R} \$ 209,60$ no espaçamento $3 \mathrm{~m} \times 3 \mathrm{~m}, \mathrm{R} \$ 315,20$ em $3 \mathrm{~m} \times 2 \mathrm{~m}$, e 379,20 em $2 \mathrm{~m} \times 2,5 \mathrm{~m}$.

Portanto, a adubação apresentada no presente estudo demonstrou uma redução nos custos com a adubação de plantio para $T$. grandis. No entanto, há que se ressaltar a necessidade de aplicações de manutenção para readequar as concentrações dos macronutrientes. Portanto, há necessidade de desenvolver estudos para obter informações sobre a adubação de manutenção para a espécie, considerando a adubação de plantio, anteriormente realizada.

\section{CONCLUSÕES}

$\mathrm{O}$ crescimento de Tectona grandis no campo foi influenciado pela adubação com NPK, recomendando-se as doses de $40 \mathrm{~kg} \mathrm{ha}^{-1}$ de $\mathrm{N}$, $50 \mathrm{~kg} \mathrm{ha}^{-1}$ de $\mathrm{P}_{2} \mathrm{O}_{5}$ e de 50 ou $100 \mathrm{~kg} \mathrm{ha}^{-1}$ de $\mathrm{K}_{2} \mathrm{O}$ na adubação de plantio.

As concentrações de macronutrientes indicaram ser preciso a reposição de $\mathrm{N}$ anteriormente aos 360 dias e maior necessidade de $\mathrm{N}$ e de $\mathrm{P}$ nos primeiros 90 dias de crescimento da espécie. 
VIEIRA, C.R.; WEBER, O.L. dos S.; SCARAMUZZA, J.F. Fertilização NPK no crescimento da teca.

\section{REFERÊNCIAS BIBLIOGRÁFICAS}

ASSOCIAÇÃO BRASILEIRA DE PRODUTORES DE FLORESTAS PLANTADAS - ABRAF. Anuário estatístico da ABRAF. Brasília, DF, 2012. 149 p.

BEHLING, M. Nutrição, partição de biomassa e crescimento de povoamentos de teca em Tangará da Serra - MT. 2009. 156 f. Tese (Doutorado em Agronomia) - Programa de Pós-graduação em Solos e Nutrição de Plantas, Universidade Federal de Viçosa, Viçosa-MG.

CRUZ, C.A.F. et al. Efeito de diferentes níveis de saturação por bases no desenvolvimento e qualidade de mudas de ipê-roxo (Tabebuia impetiginosa (Mart.) Standley). Scientia Forestalis, v. 66, p. 100-107, 2004.

EMPRESA BRASILEIRA DE PESQUISA AGROPECUÁRIA - EMBRAPA. Manual de métodos de análise de solo. 2. ed. Rio de Janeiro: Embrapa - Centro Nacional de Pesquisa de Solos, 1997. 212 p.

FARIA, M.P. Resposta de leguminosas arbóreas ao fósforo e fungo micorrízico em Latossolo Vermelho-Escuro da região Campos das Vertentes (MG). 1993. 100 f. Dissertação (Mestrado em Solos e Nutrição de Plantas) Escola Superior de Agricultura de Lavras, Universidade Federal de Lavras, Lavras.

FAVARE, L.G. Doses crescentes de nitrogênio, fósforo, potássio e diferentes níveis de saturação por bases em relação ao desenvolvimento $\mathrm{e}$ nutrição mineral de teca (Tectona grandis L.F.), sob condições de vaso. 2010. 97 f. Dissertação (Mestrado em Ciência Florestal) - Faculdade de Ciências Agronômicas, Universidade Estadual Paulista "Júlio de Mesquita Filho", Botucatu.

Adubação fosfatada e potássica em teca sob condições de campo. 2013. 53 f. Tese (Doutorado em Ciência Florestal) - Faculdade de Ciências Agronômicas, Universidade Estadual Paulista "Júlio de Mesquita Filho", Botucatu.
FAVARE, L.G.; GUERRINI, I.A.; BACKES, C. Níveis crescentes de saturação por bases e desenvolvimento inicial de teca em um Latossolo de textura média. Ciência Florestal, v. 22, n. 4, p. 693-702, 2012.

FEDERAÇÃO DA AGRICULTUA E PECUÁRIA DO ESTADO DE MATO GROSSO - FAMATO. Diagnóstico de florestas plantadas do Estado de Mato Grosso. Cuiabá: Instituto Mato-Grossense de Economia Agropecuária - IMEA, 2013. 106 p.

FIGUEIREDO, E.O; SÁ, C.P. Estimativa de custos e coeficientes técnicos para instalação e manejo de povoamentos de teca (Tectona grandis L.f.). Rio Branco: Embrapa Acre, 2005. 16 p. (Circular Técnica, n. 48).

GONÇALVES, J.L.M. et al. Produção de mudas de espécies nativas: substrato, nutrição, sombreamento e fertilização. In: GONÇALVES, J.L.M.; BENEDETTI, V. (Ed.). Nutrição e fertilização florestal. Piracicaba: IPEF, 2000. p. 310-350.

et al. Capacidade de absorção e eficiência nutricional de algumas espécies arbóreas tropicais. In: CONGRESSO NACIONAL SOBRE ESSÊNCIAS NATIVAS, 2., 1992, São Paulo. Anais... São Paulo: UNIPRESS, 1992. p. 463-469. (Revista do Instituto Florestal, v. 4, pt. 2, p. 463-469, 1992, Edição Especial).

GUNAGA, R.P.; KANFADE, A.H.; VASUDEVA, R. Soil fertility status of 20 seed production areas of Tectona grandis Linn. F. in Karnataka, India. Journal of Forest Science, v. 57, n. 11, p. 483-490, 2011.

LIMA, H.N. Crescimento inicial de sete espécies arbóreas nativas em resposta à adubação com NPK a campo. 1995. $53 \mathrm{f}$. Dissertação (Mestrado em Ciências Florestais) Departamento de Ciências Florestais, Universidade Federal de Lavras, Lavras.

MALAVOLTA, E.; VITTI, G.C.; OLIVEIRA, S.A. Avaliação do estado nutricional das plantas: princípios e aplicações. 2. ed. Piracicaba: POTAFOS, 1997. 319 p. 
VIEIRA, C.R.; WEBER, O.L. dos S.; SCARAMUZZA, J.F. Fertilização NPK no crescimento da teca.

MARKEWITZ, D. et al. Soil and tree response to $\mathrm{P}$ fertilization in a secondary tropical Forest supported by an Oxisol. Biology and Fertility of Soils, v. 48, n. 6, p. 666-678, 2012.

MARSCHNER, H. Mineral nutrition of higher plants. London: Academic Press, 1995. 889 p.

MOYA, R. et al. Efeito das propriedades físicas e químicas do solo em algumas propriedades da madeira de teca (Tectona grandis). Revista Árvore, v. 34, n. 6, p. 1109-1118, 2010.

MOYA, J.F. et al. Nutrient concentration age dynamics of teak (Tectona grandis L. f.) plantations in Central America. Forest Systems, v. 22, n. 1, p. 123-133, 2013.

MUTANAL, S.M.; PRABHAKAR, A.S.; NADAGOUDAR, B.S. Fertigation and its influence on biomass and nutrient uptake in teak. Journal of Agricultural Science, v. 13, n. 3, p. $670-675,2000$.

NICOLOSO, F.T. et al. Nutrição mineral de mudas de grápia (Apuleia leiocarpa) em Argissolo vermelho distrófico arênico: (1) efeito da adubação NPK no crescimento. Ciência Rural, v. 31, n. 6, p. 991-998, 2001.

PELISSARI, A.L.; CALDEIRA, S.F.; DRESCHER, R. Desenvolvimento quantitativo e qualitativo de Tectona grandis L.f. em Mato Grosso. Floresta e Ambiente, v. 10, n. 3, p. 371-383, 2013.

PINTO, M.L. Propriedades e características da madeira de teca (Tectona grandis) em função da idade. 2007. 124 f. Tese (Doutorado em Engenharia Civil) - Centro Tecnológico, Universidade Federal de Santa Catarina, Florianópolis.

PRADO, R.M.; VIDAL, A. Efeito da omissão de macronutrientes em solução nutritiva sobre o crescimento e nutrição do milheto. Pesquisa Agropecuária Tropical, v. 38, n. 3, p. 208-214, 2008.

PRASAD, R.; SAHA, K.; BHANDARI, A.S. Fertilizer trails in 10 and 20 year old teak plantations in Madhya Pradesh. Journal of Tropical Forestry, v. 2, n. 1, p. 15-20, 1986.
RIBEIRO, F.A. et al. Efeitos da adubação de plantio sobre o estabelecimento de mudas de Tectona grandis L.f. (teca). Revista Científica Eletrônica de Engenharia Florestal, ano 4, n. 7, 2006.

TONINI, H.; COSTA, M.C.G.; SCHWENGBER, L.A.M. Crescimento da teca (Tectona grandis) em reflorestamento na Amazônia Setentrional. Pesquisa Florestal Brasileira, n. 59, p. 5-14, 2009. 